\section{O que os dados nacionais indicam sobre a oferta e a realização de aborto previsto em lei no Brasil em 2019?}

\author{
What do the national data say about \\ the supply and performace of legal abortions \\ in Brazil in 2019?
}

\section{¿Qué indican los datos nacionales sobre oferta e interrupción del embarazo previstas por ley en Brasil en 2019?}

Marina Gasino Jacobs 1

Alexandra Crispim Boing 1

doi: 10.1590/0102-311X00085321

\section{Resumo}

Nos casos previstos em lei, o aborto é ofertado pelo Sistema Único de Saúde (SUS). O presente estudo busca mapear e caracterizar a oferta e realização do procedimento no Brasil em 2019. Foram incluídos os Serviços de Referência para Interrupção de Gravidez em Casos Previstos em Lei (SRIGCPL) registrados no Sistema do Cadastro Nacional de Estabelecimentos de Saúde (SCNES) e os estabelecimentos com registros de aborto por razões médicas e legais no Sistema de Informações Ambulatoriais ou no Sistema de Informações Hospitalares. Os estabelecimentos foram caracterizados em tipo e subtipo, natureza jurídica e convênios, e georreferenciados a partir dos dados do SCNES. Em seguida, os municípios foram divididos entre os com e os sem oferta em 2019 e então apresentados por categorias de Índice de Desenvolvimento Humano Municipal (IDH-M) e porte populacional. Logo foi calculada a taxa de realização de aborto previsto em lei dos dois grupos de municípios. Ao todo, 290 estabelecimentos ofertavam o serviço, sendo 101 SRIGCPL e 251 estabelecimentos com registro de procedimento. Os estabelecimentos estavam em 3,6\% (200) dos municipios brasileiros. A oferta se deu majoritariamente em hospitais (98,6\%), pela administração pública (62,1\%), conveniada ao SUS (99,7\%), em municípios da Região Sudeste (40,5\%), com mais de 100 mil habitantes (59,5\%) e de IDH-M alto ou muito alto (77,5\%). A taxa de realização de aborto previsto em lei entre as residentes em idade fértil dos municípios sem oferta do serviço foi de 4,8 vezes menor que nos municípios com o serviço. A oferta do aborto previsto em lei no Brasil se dá de forma desigual no território, com possível implicação no acesso ao serviço.

Acesso aos Serviços de Saúde; Aborto Legal; Equidade no Acesso aos Serviços de Saúde; Serviços de Saúde Reprodutiva

\author{
Correspondência \\ M. G. Jacobs \\ Programa de Pós-graduação em Saúde Coletiva, Centro de \\ Ciências da Saúde, Universidade Federal de Santa Catarina. \\ Campus Universitário Reitor João David Ferreira Lima, \\ Rua Delfino Conti s/ $n$, bloco H, Florianópolis, SC \\ 88040-900, Brasil. \\ marina.gjacobs@gmail.com \\ 1 Programa de Pós-graduação em Saúde Coletiva, Universidade \\ Federal de Santa Catarina, Florianópolis, Brasil.
}




\section{Introdução}

Com a Constituição Federal de 1988 e a criação do Sistema Único de Saúde (SUS), a saúde é entendida como de acesso universal, integral e igualitário, e como um dever do Estado. Entre os serviços de saúde a serem disponibilizados pelo SUS está o aborto nas situações previstas em lei. Ainda que o aborto seja criminalizado no país, ele não é punível em situações específicas. São apresentados no Código Penal como não puníveis os abortos provocados por médico quando não há outra forma de salvar a vida da gestante ou em gestações decorrentes de estupro 1. Ademais, com a Arguição de Descumprimento de Preceito Fundamental 54 julgada pelo Supremo Tribunal Federal (STF) em 2012, a interrupção de gestações de fetos anencéfalos deixa de ser tipificada como aborto, portanto não é punível 2. Além dessas situações, abortos podem ser autorizados judicialmente.

O acesso aos serviços de saúde depende de sua disponibilidade e oferta em volume suficiente. A oferta de aborto previsto em lei passou a ser normatizada pelo Ministério da Saúde em 1999 3. Dez anos depois, em 2009, eram listados pelo Ministério da Saúde 60 estabelecimentos com a oferta de aborto previsto em lei. Em 2013, essa lista foi atualizada com as Secretarias Estaduais de Saúde e a Área Técnica de Saúde da Mulher do Ministério da Saúde, quando havia 68 estabelecimentos em funcionamento com oferta de aborto previsto em lei, entretanto, apenas 37 realizavam de fato a intervenção 4 .

Ainda que o acesso universal e integral esteja no horizonte do sistema de saúde, na prática apresentam-se limites à acessibilidade, como fragmentação da rede, indisponibilidade de serviços em tempo adequado e assimetrias regionais 5. Há mais disponibilidade de serviços que dependem de maior densidade tecnológica em capitais e regiões metropolitanas, com ênfase às regiões Sul e Sudeste, sendo, portanto, mais acessíveis nessas áreas a atenção secundária e terciária do que em outras partes do país 5,6 .

Em 2014, com a Portaria no 485 do Ministério da Saúde, foram normatizados os Serviços de Referência para Interrupção de Gravidez nos Casos Previstos em Lei (SRIGCPL). Esses serviços podem ser organizados em hospitais gerais, maternidades, prontos-socorros, Unidades de Pronto-Atendimento e serviços de urgência não hospitalares com funcionamento 24 horas do dia e 7 dias da semana. Ao mesmo tempo, no país o medicamento utilizado para indução do aborto (misoprostol) é de uso exclusivo hospitalar 7 . Já os métodos cirúrgicos são aspiração manual intrauterina, também de oferta hospitalar, e curetagem uterina, que pode ter oferta ambulatorial ou hospitalar 8 . Dessa forma, a oferta de aborto previsto em lei termina também atravessada pela concentração espacial de serviços de maior densidade tecnológica.

Com a finalidade de informar diretamente quem estivesse buscando acessar o aborto previsto em lei, em 2019, uma organização não-governamental mapeou os estabelecimentos que realizavam o procedimento. O mapa foi feito a partir da lista de SRIGCPL e dos registros do Sistema de Informações Hospitalares (SIH) de 2017, ademais, por contato telefônico a organização confirmou a oferta. Não foram incluídos os registros ambulatoriais, nem os estabelecimentos que fizeram menos de duas internações no ano. Por outro lado, todas as internações com CID O04 (Aborto por razões médicas e legais), da Classificação Internacional de Doenças - 10a revisão (CID-10), foram incluídos, mesmo aquelas com procedimentos incompatíveis com a interrupção da gestação. A organização encontrou 173 estabelecimentos, dos quais 76 confirmaram realizar o aborto previsto em lei 9 .

A pouca disponibilidade do serviço no território acarreta barreiras geográficas de acesso. A oferta interage ainda com fatores financeiros, organizacionais e socioculturais 10, portanto, a escassez de oferta atinge de forma distinta cada grupo populacional, podendo privar do cuidado determinadas pessoas 5,10 .

Por sua vez, o não acesso ao aborto previsto em lei viola o direito a saúde, a tratamento digno e a liberdade. O não acesso sujeita as pessoas a situações degradantes quando são obrigadas a gestar fetos sem perspectiva de vida extrauterina, a levar a termo gestações fruto de violência, ou que as colocam em risco. Também degradante é que como única alternativa lhes reste interromper essas gestações de forma clandestina - sob a ameaça da criminalização - e potencialmente insegura, colocando sua saúde e vida em risco. Nesse sentido, ainda cabe mencionar que o aborto foi a terceira causa direta de morte materna em 2018 no Brasil 11. A razão de mortalidade materna é um indicador em que o Brasil 
tem tido avanços insuficientes nas últimas décadas e reflete a forma como o país lida com a saúde e os direitos reprodutivos das mulheres 12 .

Ainda que as exceções de punibilidade ao aborto tenham mais de 80 anos ${ }^{1}$ e as primeiras normativas de oferta do serviço no SUS mais de $20^{3}$, há pouca informação sobre a disponibilização desse serviço no território e como tem se dado a efetivação de acesso no país 13. Há uma única publicação acadêmica sobre os serviços de aborto legal no país, a qual apresenta de forma agregada a distribuição dos serviços em 2013 4,13. Soma-se a ela, a iniciativa, também mencionada, da organização não-governamental que mapeou os serviços disponíveis, com dados de 2017 9. Dessa forma, o presente estudo busca lapidar e atualizar o mapeamento da oferta e realização do aborto previsto em lei no Brasil, considerando os serviços de referência e os procedimentos de interrupção realizados em internações hospitalares ou atendimentos ambulatoriais. Além do mapeamento, são caracterizados os estabelecimentos e os municípios que ofertam o serviço, assim como apresentadas as taxas de realização de aborto previsto em lei segundo a oferta.

\section{Métodos}

Foi realizado um estudo descritivo da localização dos estabelecimentos com oferta de aborto previsto em lei em 2019 no Brasil. Para tanto, foram considerados dois grupos de estabelecimentos com oferta do procedimento: os SRIGCPL e os estabelecimentos com registros de aborto por razões médicas e legais no Sistema de Informações Ambulatoriais (SIA) ou SIH.

Como SRIGCPL foram considerados os estabelecimentos registrados em dezembro de 2019 no Sistema do Cadastro Nacional de Estabelecimentos de Saúde (SCNES), sob o código 165 (Serviços de Atenção às Pessoas em Situação de Violência Sexual) e classificação 006 (Serviços de Referência para Interrupção de Gravidez em Casos Previstos em Lei), conforme estabelecido na Portaria no 485/201414. O Cadastro Nacional de Estabelecimentos de Saúde (CNES) reúne as informações de todos os estabelecimentos de saúde no país, independentemente de sua natureza jurídica ou vinculação ao SUS. O cadastramento e a atualização dos dados são obrigatórios para que qualquer estabelecimento esteja autorizado a funcionar 15. Os arquivos de dados são disponibilizados na página de Internet do Departamento de Informática do SUS (DATASUS; https://datasus.saude.gov.br/), do Ministério da Saúde.

Como estabelecimentos com registros no SIA ou SIH de aborto por razões médicas e legais em 2019, foram incluídos aqueles que realizaram atendimentos ambulatoriais ou internações com diagnóstico principal CID O04 (Aborto por razões médicas e legais) e os procedimentos de interrupção de gestação compatíveis segundo a Tabela de Procedimentos, Medicamentos, Órteses, Próteses e Materiais Especiais (OPM) do SUS, a saber: "Curetagem pós-abortamento/puerperal" e "Esvaziamento uterino pós-aborto por AMIU” 8. O SIA e o SIH têm como finalidade principal o financiamento dos serviços, dessa forma, neles constam todos os atendimentos ambulatoriais e internações (respectivamente) pagas pelo SUS, sejam na rede pública ou privada. Os arquivos de dados também são disponibilizados na página de Internet do DATASUS (https://datasus.saude.gov.br/), assim como os arquivos auxiliares e o programa TabWin para tabulação.

Nos registros de atendimentos ambulatoriais do SIA e internações do SIH é especificado o código de CNES do estabelecimento que faz o atendimento. No SCNES, os SRIGCPL também são apresentados por seu código CNES. A partir do código CNES dos estabelecimentos registrados nesses três sistemas, foram coletadas no SCNES as seguintes informações sobre cada estabelecimento: tipo e subtipo de unidade, natureza jurídica, convênios, CEP, bairro, município e Unidade da Federação (UF) de localização.

Os endereços dos estabelecimentos foram georreferenciados com o software QGIS3.4 (https:// qgis.org/en/site/) por meio do API HQGIS da Plataforma Here (https://www.here.com/). Inicialmente os locais foram buscados por Código de Endereçamento Postal (CEP). Nas situações em que o CEP não foi encontrado, ou o CEP encontrado não foi condizente ao município de localização do estabelecimento, o ponto foi buscado por bairro, município e UF.

Os pontos encontrados foram então sobrepostos às malhas digitais do Brasil com divisão político-administrativa vigente no Censo Demográgico de 2010 disponibilizadas pelo Instituto Brasileiro de Geografia e Estatística (IBGE; https://censo2010.ibge.gov.br/), adotando o Sistema de Referência 
Geocêntrico para as Américas (SIRGAS 2000; https://www.sirgas.org/pt/). Todos os registros em áreas dentro do Distrito Federal foram atribuídos a Brasília, único município da UF reconhecido pelo IBGE.

Após o georreferenciamento dos estabelecimentos, foi feito um descritivo dos municípios brasileiros conforme a presença de estabelecimentos com oferta de aborto previsto em lei em 2019. Os municípios do país foram divididos em dois grupos, aqueles em que houve oferta de aborto previsto em lei e aqueles em que não houve. Foram apresentadas as frequências absolutas e relativas para as categorias de Índice de Desenvolvimento Humano Municipal (IDH-M) e de porte populacional de cada grupo. O Índice de Desenvolvimento Humano (IDH) é uma medida síntese das condições básicas de vida de uma localidade e é composto pelos pilares: oportunidade de levar uma vida longa e saudável, acesso ao conhecimento, e possibilidade de desfrutar de um padrão de vida digno 16 .

Os indicadores utilizados para compor o IDH-M são: expectativa de vida ao nascer, fluxo escolar da população jovem, escolaridade da população adulta e renda municipal per capita. O índice varia de 0 a 1 e é classificado da seguinte forma: muito baixo 0,000-0,499; baixo 0,500-0,599; médio 0,600-0,699; alto 0,700-0,799, e muito alto 0,800-1,000. O IDH-M apresentado pelo Atlas do Desenvolvimento Humano no Brasil, ainda que publicado em 2013, tem como base os dados do Censo Demográfico de 2010 16. O índice foi utilizado mesmo que seus dados tenham sido coletados 9 anos antes do período abordado no presente estudo, pois é capaz de oferecer um panorama dos municípios no conjunto. Já o porte populacional teve como fonte as estimativas populacionais do IBGE para 2019 e foi apresentado nas categorias: até 5.000 habitantes; de 5.001 a 10.000; de 10.001 a 20.000; de 20.001 a 50.000; de 50.001 a 100.000; de 100.001 a 500.000 ; e mais de 500.000 habitantes.

Em seguida, foram calculadas as taxas de realização de aborto previsto em lei entre as residentes dos dois grupos de municípios (com e sem oferta de aborto previsto em lei). As taxas foram calculadas por meio da razão dos registros de aborto por razões médicas e legais em cada um dos dois grupos de municípios pela população feminina de 10 a 49 anos residente naqueles mesmos dois grupos. Para tanto, foram utilizados os registros de atendimento ambulatorial disponíveis no SIA e de internações disponíveis no SIH com diagnóstico principal CID O04 (Aborto por razões médicas e legais), de mulheres em idade fértil (10 a 49 anos) que realizaram procedimentos de interrupção de gestação em 2019. Foram utilizadas as estimativas das populações municipais por sexo e faixa etária em 2019 elaboradas pelo Ministério da Saúde e disponibilizadas pelo DATASUS.

O estudo usa apenas informações de acesso público e não inclui dados de identificação ou identificáveis dos usuários, dispensando a submissão ao Comitê de Ética, conforme a Resolução no 510/2016 do Conselho Nacional de Saúde.

\section{Resultados}

Havia 101 estabelecimentos registrados como SRIGCPL em 2019. Foram encontrados também 251 estabelecimentos com registros de aborto por razões médicas e legais, fossem ambulatoriais ou hospitalares, naquele ano. Conforme apresentado nas Figuras 1 e 2, havia SRIGCPL sem abortos por razões médicas e legais registrados e serviços com o procedimento registrado que não eram SRIGCPL. Ao todo, foram 290 estabelecimentos com oferta do serviço.

Dos 251 estabelecimentos que fizeram algum aborto por razões médicas e legais em 2019, 62 $(24,7 \%)$ estavam registrados no SCNES como SRIGCPL. Por outro lado, dos 101 estabelecimentos registrados como SRIGCPL no SCNES, 39 (38,6\%) não realizaram qualquer procedimento naquele ano (Figura 1).

Dos 101 SRIGCPL, 96 (95\%) eram hospitais, sendo 70 hospitais gerais, 25 maternidades e 1 hospital pediátrico. Além desses, estavam registradas 2 policlínicas, 1 centro de especialidade, 1 pronto atendimento e 1 unidade mista. No que se refere aos 251 estabelecimentos com algum procedimento realizado, todos eram hospitais, $201(80,1 \%)$ hospitais gerais, $48(19,1 \%)$ hospitais especializados sendo 45 maternidades, 1 pediátrico e 2 não especificados -, os últimos $2(0,8 \%)$ eram prontos-socorros gerais, também em hospitais. 
Oferta de aborto previsto em lei entre Serviços de Referência para Interrupção de Gravidez em Casos Previstos em Lei (SRIGCPL) e estabelecimentos com registro de aborto por razões médicas e legais. Brasil, 2019.

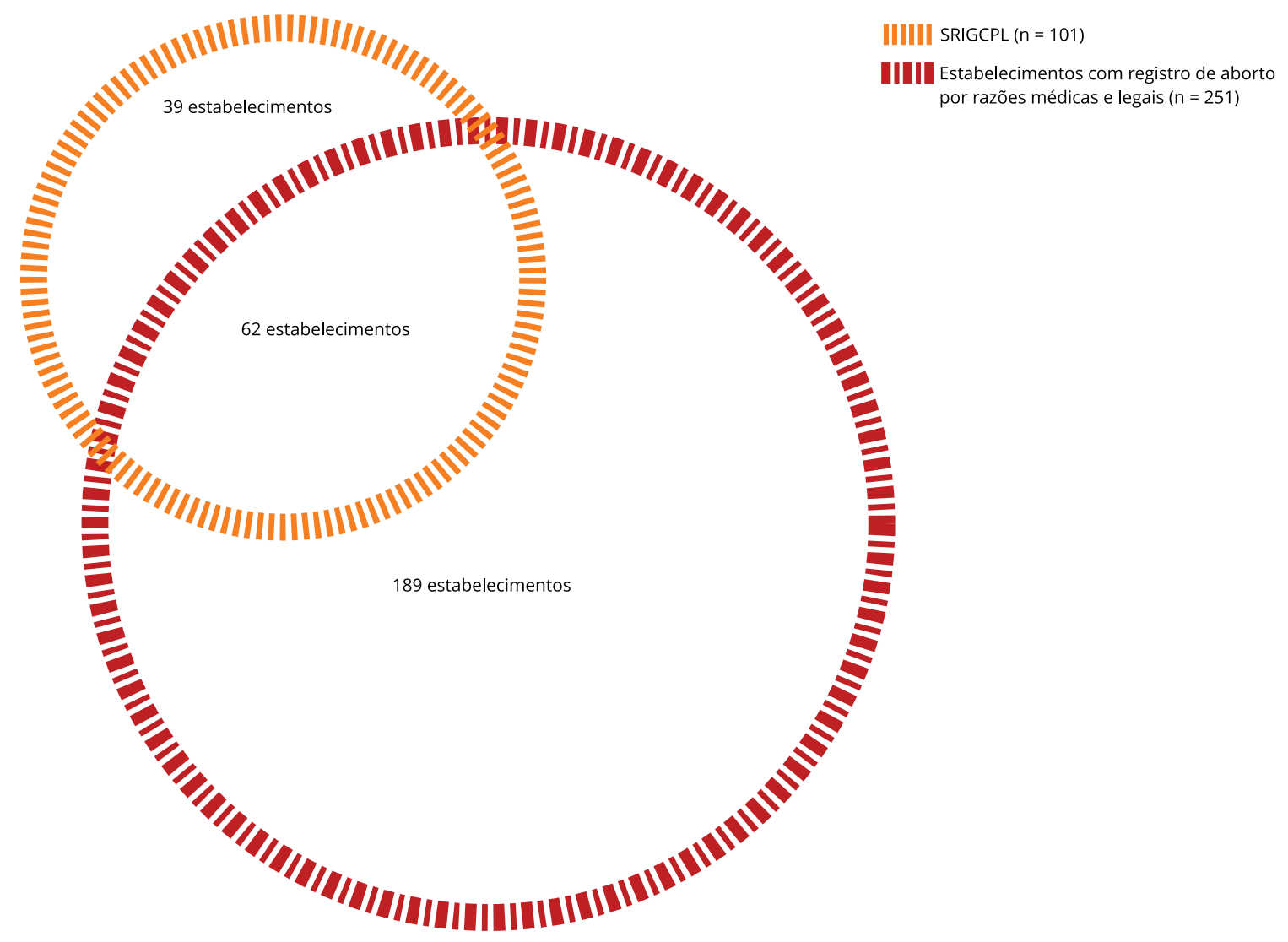

Fonte: Sistema de Informações Ambulatoriais e Sistema de Informações Hospitalares/Ministério da Saúde (https://datasus.saude.gov.br/).

Quanto à natureza jurídica dos estabelecimentos, dos 101 SRIGCPL, 80 (79,2\%) correspondiam à administração pública, 8 (7,9\%) a entidades empresariais e 13 (12,9\%) a entidades sem fins lucrativos. De todos os serviços cadastrados como SRIGCPL, apenas um não tinha convênio com o SUS. Dos 251 estabelecimentos que realizaram abortos previstos em lei, 150 (60\%) tinham administração pública, 9 $(3,6 \%)$ eram entidades empresariais e 92 (36,8\%) entidades sem fins lucrativos. Como aqui trata-se de procedimentos registrados no SIA e SIH, portanto, financiados pelo SUS, todos os estabelecimentos eram conveniados ao sistema. Destaca-se que das entidades empresariais registradas, apenas duas não eram empresas públicas.

Os 290 estabelecimentos com oferta de aborto previsto em lei em 2019 distribuem-se em apenas 3,6\% (200) dos municípios brasileiros, nenhum deles no Estado do Amapá (Figura 2; Tabela 1). Ainda assim, entre os municípios da Região Norte, 6,4\% ofertavam o serviço, o maior percentual entre as regiões (Tabela 1), contudo, o Sudeste concentrou 40,5\% do total de municípios com oferta.

O percentual de municípios com oferta de aborto previsto em lei foi mais alto entre aqueles de maiores IDH-M e porte. Não houve qualquer oferta em municípios de IDH-M muito baixo, tampouco naqueles de até 10 mil habitantes (Tabela 1). A oferta se deu majoritariamente em municípios com IDH-M alto ou muito alto (77,5\%) e com mais de 100 mil habitantes $(59,5 \%)$. 
Figura 2

Localização dos Serviços de Referência para Interrupção de Gravidez em Casos Previstos em Lei (SRIGCPL) e dos estabelecimentos com registro de interrupções legais de gestação. Brasil, 2019.

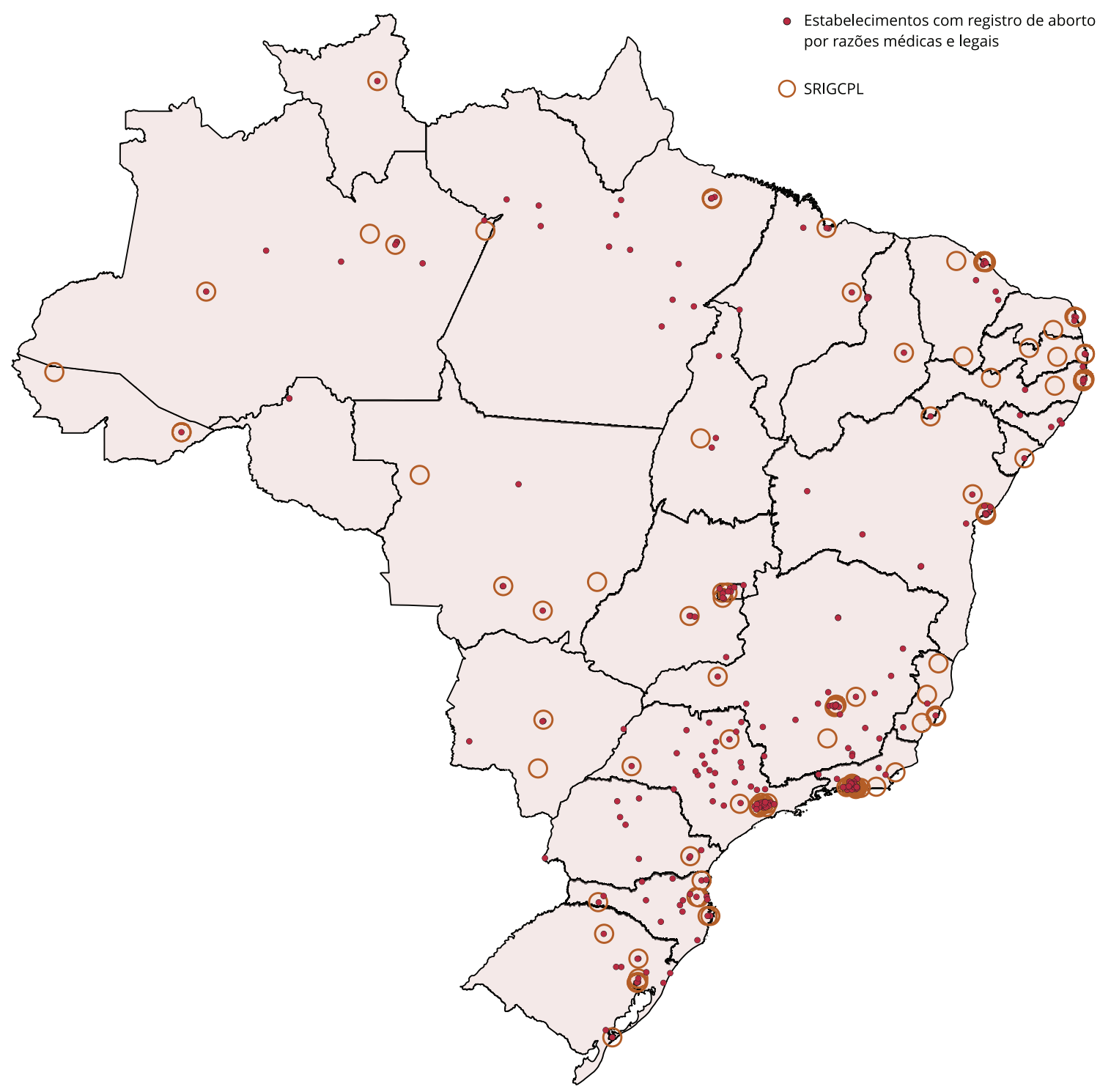

Fonte: Sistema de Informações Ambulatoriais e Sistema de Informações Hospitalares/Ministério da Saúde (https://datasus.saude.gov.br/).

Ainda que os municípios com a oferta de aborto previsto em lei tenham maior porte, são em menor número e, por isso, não concentram a maioria da população que pode precisar de um aborto previsto em lei. Calcula-se que em 2019, 58,3\% (37.590.491) das mulheres em idade fértil viviam em municípios em que o aborto previsto em lei não era ofertado, enquanto as outras 41,7\% (26.880.028) viviam em municípios com a oferta do serviço. Ainda comparando os grupos de municípios, a taxa de realização de aborto previsto em lei entre as residentes em idade fértil dos municípios sem oferta do serviço em 2019 foi de 1,1 a cada 100 mil; já nos municípios com oferta de aborto previsto em lei a taxa foi de 5,3 por 100 mil mulheres em idade fértil (10 a 49 anos). 


\section{Tabela 1}

Oferta de aborto previsto em lei nos municípios brasileiros por estratos de desenvolvimento humano, porte populacional e por região, 2019.

\begin{tabular}{|c|c|c|}
\hline \multirow[t]{2}{*}{ Variável municipal } & \multicolumn{2}{|c|}{ Oferta de aborto previsto em lei [n (\%)] } \\
\hline & Sim & Não \\
\hline \multicolumn{3}{|l|}{ IDH-M * } \\
\hline Muito baixo & $0(0,0)$ & $32(100,0)$ \\
\hline Baixo & $11(0,8)$ & $1.354(99,2)$ \\
\hline Médio & $34(1,5)$ & $2.197(98,5)$ \\
\hline Alto & $131(6,9)$ & $1.758(93,1)$ \\
\hline Muito alto & $24(54,5)$ & $20(45,5)$ \\
\hline Não classificados & $0(0,0)$ & $9(100,0)$ \\
\hline \multicolumn{3}{|c|}{ Porte populacional (habitantes) } \\
\hline Até 5.000 & $0(0,0)$ & $1.253(100,0)$ \\
\hline $5.001-10.000$ & $0(0,0)$ & $1.199(100,0)$ \\
\hline $10.001-20.000$ & $6(0,4)$ & $1.338(99,6)$ \\
\hline $20.001-50.000$ & $32(2,9)$ & $1.069(97,1)$ \\
\hline $50.001-100.000$ & $43(12,3)$ & $306(87,7)$ \\
\hline $100.001-500.000$ & $78(28,3)$ & $198(71,7)$ \\
\hline Mais de 500.000 & $41(85,4)$ & $7(14,6)$ \\
\hline \multicolumn{3}{|l|}{ Região } \\
\hline Norte & $29(6,4)$ & $421(93,6)$ \\
\hline Nordeste & $38(2,1)$ & $1.756(97,9)$ \\
\hline Sudeste & $81(4,9)$ & $1.587(95,1)$ \\
\hline Sul & $38(3,2)$ & $1.153(96,8)$ \\
\hline Centro-oeste & $14(3,0)$ & $453(97,0)$ \\
\hline Total de municípios & $200(3,6)$ & $5.370(96,4)$ \\
\hline
\end{tabular}

IDH-M: Índice de Desenvolvimento Humano Municipal.

Fonte: Sistema de Informações Ambulatoriais e Sistema de Informações Hospitalares/Ministério da Saúde (https://datasus.saude.gov.br/); Programa das Nações Unidas para o Desenvolvimento; Instituto de Pesquisa Econômica Aplicada; Fundação João Pinheiro 16.

* IDH-M: muito baixo 0,000-0,499; baixo 0,500-0,599; médio 0,600-0,699; alto 0,700-0,799, e muito alto 0,800-1,000.

\section{Discussão}

Inicialmente, fica evidenciada a incompatibilidade entre os registros de realização de aborto previsto em lei e os SRIGCPL: a maior parte dos serviços que realizaram algum aborto não estavam registrados como SRIGCPL e mais de 1/3 dos SRIGCPL não realizaram nenhum procedimento no ano. Como visto, a realização de aborto previsto em lei não é condicionada ao registro do estabelecimento como SRIGCPL, todavia o cadastro tem a finalidade de fornecer informações, o que cabe à gestão do estabelecimento e não depende de habilitação prévia, mas não gera incentivo financeiro específico. Destaca-se que, ainda que haja SRIGCPL em estabelecimentos não hospitalares, todos os procedimentos realizados naquele ano se deram em hospitais. Também chama atenção o registro como SRIGCPL de apenas um estabelecimento sem convênio com o SUS, o que poderia indicar a deficiência do registro, ou a quase exclusividade de financiamento de aborto previsto em lei pelo sistema público.

O descompasso entre os registros dificulta a consolidação da informação para monitoramento e avaliação da oferta do serviço pela gestão e pela academia, assim como atrapalha o acesso à informação pelos usuários. Por meio de transparência ativa, os serviços listados pelo Ministério da Saúde são apenas os cadastrados no SCNES como SRIGCPL 17. Mesmo essa informação não é acessível para o cidadão comum, pois demanda conhecimento da nomenclatura específica utilizada pelo Ministério da Saúde 18. A inacessibilidade da informação também se fez ver em levantamento feito em 2018, 
que constatou que apenas 2 das 27 UF disponibilizavam nos sites de seus órgãos de saúde a lista dos estabelecimentos com oferta de aborto previsto em lei; das 26 capitais de estado, apenas 6 ofereciam a informação 18 .

Nesse sentido, a existência dos SRIGCPL que não realizaram qualquer aborto previsto em lei em 2019, além de falha de registro, pode indicar a presença de barreiras de acesso que estejam além da barreira geográfica, como o desconhecimento sobre a previsão do aborto nos casos excepcionais, sua oferta no SUS e os serviços que o realizam 9,18,19, pode apontar, ainda, medo da criminalização, ou mesmo vergonha pelo estigma do procedimento 20,21. Além disso, são relatadas barreiras organizacionais, como a exigência de Boletim de Ocorrência, laudo do Instituto Médico-Legal (IML) ou alvará judicial 4,9; recusa dos profissionais de saúde em realizar o procedimento 22,23,24,25; e negativas por suspeição à palavra de quem busca por cuidado 26. É possível também que os estabelecimentos simplesmente não ofertem o serviço, ainda que estejam cadastrados para tanto. Essa não oferta foi constatada em 2013, quando uma pesquisa encontrou que apenas 37 dos 68 estabelecimentos cadastrados no Ministério da Saúde para oferta do aborto previsto em lei de fato ofertavam o serviço 4 . Naquele momento, entretanto, não haviam sido instituídos os SRIGCPL 14. Mais recentemente, em 2019, um levantamento encontrou ao menos 13 SRIGCPL que em contato telefônico declararam não realizar o procedimento ${ }^{9}$. Por fim, vale apresentar que um dos SRIGCPL cadastrados é uma cooperativa não conveniada ao SUS, portanto, apenas nesse caso é natural que não haja registro de procedimento realizado no estabelecimento e financiado pelo sistema.

Pode ser destacado que o estabelecimento ter realizado de algum aborto previsto em lei naquele ano, não significa a oferta sustentada do serviço, ademais, nem todo estabelecimento com oferta abrange as três causas previstas no país 4,24. Merece atenção o fato de que em casos de risco de morte da gestante não é possível o estabelecimento ou o médico recusar-se a realizar a interrupção da gestação quando tem condições de prestar o atendimento 27 . Portanto, nas gestações decorrentes de estupro e nos casos de anencefalia as barreiras e objeções podem se fazer mais presentes 25 .

Em que pese as limitações de registro, a oferta do aborto previsto em lei se deu em 3,6\% dos municípios em 2019. Mesmo a Região Norte, com a maior proporção, teve apenas 6,4\% de seus municípios com oferta do serviço, o que naquela região é um desfio adicional de acesso dadas as longas distâncias entre sedes municipais e as dificuldades de transporte. Está na Região Norte a única UF sem nenhum serviço, o Amapá.

Os municípios com oferta de aborto previsto em lei estão majoritariamente na Região Sudeste, têm maior porte e maior IDH-M. Dado esse índice ser composto por indicadores de saúde, educação e renda, o achado pode apontar uma camada suplementar de dificuldade de acesso ao procedimento aos mais pobres e menos escolarizados. Esta desigualdade historicamente é documentada nas Pesquisas Nacionais por Amostra de Domicílios (PNAD) de 1998, 2003 e 2008 e Pesquisas Nacionais de Saúde (PNS) de 2013 e 2019, mostrando maior proporção de uso de serviços de saúde entre mulheres, brancas, de alta escolaridade, alta renda, ou residentes em áreas urbanas, ainda que as desigualdades de uso venham diminuindo ao longo do tempo 28,29,30,31.

Quanto às taxas de realização de aborto previsto em lei, as residentes de municípios com oferta realizaram 4,8 vezes mais abortos que as residentes em municípios sem oferta. Supondo que a necessidade de aborto previsto em lei fosse a mesma em todos os municípios, aproximadamente 4 a cada 5 mulheres que precisaram de um aborto previsto em lei e viviam em municípios que não o ofertavam deixaram de acessar o serviço. Ou seja, naquele ano 1.570 interrupções de gestação podem ter deixado de ser realizadas em municípios que não ofertavam o procedimento. Isso equivaleria a 46,2\% da necessidade total do país não sendo atendida por escassez de oferta.

O não acesso a internações hospitalares por insuficiência de oferta foi encontrado com a PNAD de 1998, apontando associação entre maior oferta de leitos hospitalares e o número de internações 32. Em 2000 foi encontrado, ainda, que a probabilidade de atendimento caía com o aumento da distância entre o local de moradia e o hospital. Mesmo distâncias de até $20 \mathrm{~km}$ provocavam redução na probabilidade de atendimento, destacando a capacidade local de internação como facilitadora do uso do serviço 33 .

Não há informação disponível sobre a redução de acesso especificamente ao aborto previsto em lei no Brasil. Entretanto, evidências de outras partes do mundo têm apontado que maiores distâncias até o serviço estão associadas à redução do acesso ao aborto 20,34,35,36,37 e é uma barreira mais dificilmente 
superável por grupos vulnerabilizados, como não-brancas $35,36,38$ ou de menor escolaridade 20,39. A fim de superar essas barreiras, vêm se desenhando estratégias a partir de evidências, da experiência de outros países e de recomendações internacionais, como utilizando-se de recursos de telessaúde 24,40, capilarizando a oferta do serviço por meio da atenção primária 39,41,42,43,44, e mesmo revendo a oferta do aborto como exclusiva de profissionais médicos 45,46,47,48,49. Essas soluções seriam valorosas no contexto brasileiro, em que todos os abortos previstos em lei foram realizados em hospitais em 2019. Para sua implantação, seria fundamental que o misoprostol deixasse de ter uso exclusivo em ambiente hospitalar 39,41,42,43,44, a aprovação da mifepristona e incorporação na Relação Nacional de Medicamentos Essenciais e nos protocolos para realização do aborto previsto em lei 24,43,44, assim como possibilitar a oferta de aspiração manual intrauterina (AMIU) como procedimento ambulatorial 8,41,43,46,48,49. Já a oferta do procedimento por outros profissionais dependeria de alteração do Código Penal brasileiro, o qual restringe a não punibilidade dos casos excepcionais aos abortos realizados por médicos 1 .

Destaca-se, entretanto, que o acesso ao aborto previsto em lei encontra barreiras para além de oferta do serviço, portanto, tem percentual de realização ainda menor. A fim de dimensionar a magnitude dessas gestações: em 2019, as Secretarias Estaduais de Segurança Pública e a Defesa Social de 21 UF registraram juntas 66.123 estupros 50 , os quais estimam-se ser pouco mais de $10 \%$ do total das ocorrências 50,51,52; das mulheres estupradas, ao menos $5 \%$ engravidam 51,53,54,55.

Quanto às outras causais que possibilitam o aborto legal, não há estimativas de percentual de realização da interrupção. São desconhecidos os itinerários das gestantes de fetos anencéfalos, no que diz respeito à possibilidade de interrupção por risco à vida da gestante, não há detalhamento sobre as situações que vêm sendo incluídas nesse critério, tampouco como tem se dado a decisão pela interrupção nesses casos 13 .

É imprescindível apontar que o não acesso ao aborto previsto em lei pelas pessoas que precisam do serviço expõe gestantes a prejuízos físicos, sociais e psicológicos por levarem a termo gestações em que não há expectativa de vida extrauterina, gestações que ameaçam suas vidas ou que são decorrentes de violência e, em seguida, por precisarem lidar com o fruto da gestação. Além de expor a gestante a tratamento degradante, as falhas de acesso podem levar a abortos clandestinos 56 e potencialmente inseguros, pois, no contexto de criminalização, não contam com informações seguras difundidas, medicamentos com qualidade verificada, atendimento por profissionais qualificados e mesmo serviços pós-abortamento $57,58,59,60$.

Pesquisar a temática do aborto no Brasil é um desafio mesmo nas situações em que ele é legal. Quanto às limitações do presente estudo, inicialmente, pode ser mencionada a possível heterogeneidade de preenchimento dos sistemas de informação que foram fonte dos dados. São sistemas utilizados em todo o território nacional, portanto, alimentados em contextos múltiplos, ainda que sigam normas únicas. Nesse sentido, tendo sido incluídos no estudo apenas os procedimentos com diagnóstico principal CID O04 (Aborto por razões médicas e legais), podem ter sido subestimados aqueles realizados em casos de risco de vida à gestante e anencefalia fetal, passíveis de registros distintos. Além disso, o aborto previsto em lei não tem código específico de procedimento, assim, de acordo com a Tabela de Procedimentos, Medicamentos e OPM do SUS são compatíveis com o diagnóstico principal CID O04, os procedimentos de interrupção “Curetagem pós-abortamento/puerperal” e "Esvaziamento uterino pós-aborto por AMIU” 8. Contudo, podem ter sido aqui subestimados os abortos previstos em lei e sua oferta em caso de registro de procedimentos de interrupção incompatíveis com o CID O04, segundo a referida tabela. O descompasso entre CID-10 e procedimentos foi momentaneamente suprimido pela Portaria no 415/2014 publicada pelo Ministério da Saúde 61, que incluía "Interrupção da gestação/antecipação terapêutica do parto previstas em lei” na Tabela de Procedimentos, Medicamentos $e$ OPM do SUS. A Portaria, entretanto, foi revogada uma semana após ser sancionada 62. Desde então, não houve outra publicação que suprisse essa lacuna, prejudicado o registro adequado do procedimento devido ao estigma, mesmo nas situações legais.

Ainda assim, o uso das informações disponíveis nos sistemas do Ministério da Saúde oferece um panorama do cenário de oferta do aborto previsto em lei no país. Fica apontado que os SRIGCPL têm, majoritariamente, administração pública e financiamento estatal. Evidencia-se que a oferta está concentrada em um percentual ínfimo de municípios, os quais têm por característica grande porte e maior IDH-M. É explicitada também grande diferença nas taxas de realização do procedimento legal entre municípios com e sem a oferta, podendo indicar lacunas de assistência, o que, mais uma vez, 
anuncia-se como violação aos direitos das pessoas que precisam do serviço e uma violência institucional, ainda que por omissão.

Nesse contexto, se faz premente, por um lado, a qualificação dos registros nos sistemas de informação em saúde no que tange o aborto previsto em lei, a fim de possibilitar o monitoramento, a avaliação e a qualificação da oferta do serviço, assim como a transparência e a informação à população. Por outro lado, urge que se recorra a estratégias de ampliação e capilarização da oferta do aborto previsto em lei para que, de forma respaldada pelos princípios do SUS, o lugar onde as pessoas vivem não impeça o acesso universal e equânime ao serviço de saúde.

\section{Colaboradores}

M. G. Jacobs contribuiu com a concepção do estudo, redação e revisão do manuscrito. A. C. Boing contribuiu com a concepção do estudo e revisão manuscrito. Ambas as autoras aprovaram a versão final a ser publicada.

\section{Informações adicionais}

ORCID: Marina Gasino Jacobs (0000-0002-24886016); Alexandra Crispim Boing (0000-0001-77924824);

\section{Agradecimento}

À Coordenação de Aperfeiçoamento de Pessoal de Nível Superior (CAPES: Código de Financiamento 001).

\section{Referências}

1. Brasil. Decreto-Lei no 2.848, de 7 de dezembro de 1940. Código Penal. Diário Oficial da União 1940; $31 \mathrm{dez}$.

2. Supremo Tribunal Federal. Arguição de Descumprimento de Preceito Fundamental no 54. Relator Ministro Marco Aurélio. Julgado em 12/04/2012. Brasília: Supremo Tribunal Federal; 2012.

3. Ministério da Saúde. Prevenção e tratamento dos agravos resultantes da violência sexual contra mulheres e adolescentes: norma técnica. Brasília: Ministério da Saúde; 1999.

4. Madeiro AP, Diniz D. Serviços de aborto legal no Brasil: um estudo nacional. Ciênc Saúde Colet 2016; 21:563-72.

5. Assis MMA, Jesus WLA. Acesso aos serviços de saúde: abordagens, conceitos, políticas e modelo de análise. Ciênc Saúde Colet 2012; 17:2865-75.

6. Solla J, Chioro A. Atenção ambulatorial especializada. In: Giovanella L, Escorel S, Lobato LVC, Noronha JC, Carvalho AI, organizadores. Políticas e sistema de saúde no Brasil. Rio de Janeiro: Editora Fiocruz/Centro Brasileiro de Estudos de Saúde; 2008. p. 627-64.

7. Ministério da Saúde. Portaria no 344, de 12 de maio de 1998. Aprova o Regulamento Técnico sobre substâncias e medicamentos sujeitos a controle especial. Diário Oficial da União 1998; 15 mai.

8. Ministério da Saúde. Sistema de Gerenciamento da Tabela de Procedimentos, Medicamentos e OPM do SUS. Tabela unificada. http://sigtap. datasus.gov.br/tabela-unificada/app/sec/ini cio.jsp (acessado em 25/Set/2020). 
9. Article 19. Acesso à informação e aborto legal: mapeando desafios nos serviços de saúde. São Paulo: Article 19; 2019.

10. Gulliford M, Figueroa-Munoz J, Morgan M, Hughes D, Gibson B, Beech R, et al. What does "access to health care" mean? J Health Serv Res Policy 2002; 7:186-8.

11. Ministério da Saúde. Audiência pública - interrupção voluntária da gravidez. Brasília: Supremo Tribunal Federal; 2018.

12. Drezett J. Mortalidade materna no Brasil. Insucesso no cumprimento do quinto Objetivo de Desenvolvimento do Milênio. Reprod Clim 2013; 28:89-91.

13. Fonseca SC, Domingues RMSM, Leal MC, Aquino EML, Menezes GMS. Aborto legal no Brasil: revisão sistemática da produção científica, 2008-2018. Cad Saúde Pública 2020; 36 Suppl 1:e00189718.

14. Ministério da Saúde. Portaria no 485, de 1o de abril de 2014. Redefine o funcionamento do Serviço de Atenção às Pessoas em Situação de Violência Sexual no âmbito do Sistema Único de Saúde (SUS). Diário Oficial da União 2014; 2 abr.

15. Ministério da Saúde. Portaria no 1.646, de 2 de outubro de 2015. Institui o Cadastro Nacional de Estabelecimentos de Saúde (CNES). Diário Oficial da União 2015; 5 out.

16. Programa das Nações Unidas para o Desenvolvimento; Instituto de Pesquisa Econômica Aplicada; Fundação João Pinheiro. Índice de Desenvolvimento Humano municipal brasileiro. Brasília: Programa das Nações Unidas para o Desenvolvimento/Instituto de Pesquisa Econômica Aplicada/Fundação João Pinheiro; 2013.

17. Ministério da Saúde. Cadastro Nacional de Estabelecimentos de Saúde. http://cnes2.datasus. gov.br/ (acessado em 10/Jun/2020).

18. Article 19. Breve panorama sobre aborto legal e transparência no Brasil. São Paulo: Article $19 ; 2018$

19. Talib RA, Pereira IG, Sá LCG, Citeli MT. Panorama do aborto legal no Brasil. São Paulo: Católicas pelo Direito de Decidir; 2006.

20. Friedman J, Saavedra-Avendaño B, Schiavon R, Alexander L, Sanhueza P, Rios-Polanco R, et al. Quantifying disparities in access to publicsector abortion based on legislative differences within the Mexico City Metropolitan Area. Contraception 2019; 99:160-4.

21. Culwell KR, Hurwitz M. Addressing barriers to safe abortion. Int J Gynaecol Obstet 2013; 121 Suppl 1:S16-9.

22. Diniz D, Madeiro A, Rosas C. Conscientious objection, barriers, and abortion in the case of rape: a study among physicians in Brazil. Reprod Health Matters 2014; 22:141-8.

23. Branco JGO, Brilhante AVM, Vieira LJES, Manso AG. Objeção de consciência ou instrumentalização ideológica? Uma análise dos discursos de gestores e demais profissionais acerca do abortamento legal. Cad Saúde Pública 2020; 36 Suppl 1:e00038219.
24. Rosas CF, Paro HBMS. Serviços de atenção ao aborto previsto em lei: desafios e agenda no Brasil. Brasília: Centro Feminista de Estudos e Assessoria/Sexuality Policy Watch; 2021.

25. Diniz D. Objeção de consciência e aborto: direitos e deveres dos médicos na saúde pública. Rev Saúde Pública 2011; 45:981-5.

26. Diniz D, Dios VC, Mastrella M, Madeiro AP. A verdade do estupro nos serviços de aborto legal no Brasil. Rev Bioét 2014; 22:291-8.

27. Conselho Federal de Medicina. Resolução CFM no 1.931/2009. Aprova o Código de Ética Médica. Diário Oficial da União 2009; 24 set.

28. Almeida G, Sarti FM, Ferreira FF, Diaz MDM, Campino ACC. Analysis of the evolution and determinants of income-related inequalities in the Brazilian health system, 1998-2008. Rev Panam Salud Pública 2013; 33:90-7.

29. Instituto Brasileiro de Geografia e Estatística. Pesquisa Nacional de Saúde, 2019. Informações sobre domicílios, acesso e utilização dos serviços de saúde. Rio de Janeiro: Instituto Brasileiro de Geografia e Estatística; 2020.

30. Macinko J, Lima-Costa MF. Horizontal equity in health care utilization in Brazil, 1998-2008. Int J Equity Health 2012; 11:33.

31. Stopa SR, Malta DC, Monteiro CN, Szwarcwald CL, Goldbaum M, Cesar CLG. Use of and access to health services in Brazil, $2013 \mathrm{Na}$ tional Health Survey. Rev Saúde Pública 2017; 51 Suppl 1:3s.

32. Castro MSM, Travassos C, Carvalho MS. Efeito da oferta de serviços de saúde no uso de internações hospitalares no Brasil. Rev Saúde Pública 2005; 39:277-84.

33. Oliveira EXG, Travassos C, Carvalho MS. Acesso à internação hospitalar nos municípios brasileiros em 2000: territórios do Sistema Único de Saúde. Cad Saúde Pública 2004; 20 Suppl 2:S298-309.

34. Grossman D, White K, Hopkins C, Potter JE. How greater travel distance due to clinic closures reduced access to abortion in Texas. PRC Research Brief 2017; 2:1-3.

35. Cunningham S, Lindo J, Myers CK, Schlosser A. How far is too far? New evidence on abortion clinic closures, access, and abortions. Cambridge: National Bureau of Economic Research; 2017. (NBER Working Paper, 23366).

36. Venator J, Fletcher J. Undue burden beyond Texas: an analysis of abortion clinic closures, births, and abortions in Wisconsin. Cambridge: National Bureau of Economic Research; 2019. (NBER Working Paper, 26362).

37. Brown BP, Hebert LE, Gilliam M, Kaestner R. Distance to an abortion provider and its association with the abortion rate: a multistate longitudinal analysis. Perspect Sex Reprod Health 2020; 52:227-34.

38. Goyal V, Brooks IHM, Powers DA. Differences in abortion rates by race-ethnicity after implementation of a restrictive Texas law. Contraception 2020; 102:109-14. 
39. Senderowicz L, Sanhueza P, Langer A. Education, place of residence and utilization of legal abortion services in Mexico City, 2013-2015. Int Perspect Sex Reprod Health 2018; 44:43-50.

40. Endler M, Lavelanet A, Cleeve A, Ganatra B, Gomperts R, Gemzell-Danielsson K. Telemedicine for medical abortion: a systematic review. BJOG 2019; 126:1094-102.

41. Maia MN. Oferta de aborto legal na atenção primária à saúde: uma chamada para ação. Rev Bras Med Fam Comunidade 2021; 16:2727.

42. Mundle S, Elul B, Anand A, Kalyanwala S, Ughade S. Increasing access to safe abortion services in rural India: experiences with medical abortion in a primary health center. Contraception 2007; 76:66-70.

43. World Health Organization. Safe abortion: technical and policy guidance for health systems. 2nd Ed. Geneva: World Health Organization; 2012.

44. World Health Organization. Medical management of abortion. Geneva: World Health Organization; 2018.

45. Barnard S, Kim C, Park MH, Ngo TD. Doctors or mid-level providers for abortion. Cochrane Database Syst Rev 2015; (7):CD011242.

46. Renner R-M, Brahmi D, Kapp N. Who can provide effective and safe termination of pregnancy care? A systematic review. BJOG 2013; 120:23-31.

47. Warriner I, Wang D, Huong NM, Thapa K, Tamang A, Shah I, et al. Can midlevel health-care providers administer early medical abortion as safely and effectively as doctors? A randomised controlled equivalence trial in Nepal. Lancet 2011; 377:1155-61.

48. Ngo TD, Park MH, Free C. Safety and effectiveness of termination services performed by doctors versus midlevel providers: a systematic review and analysis. Int $J$ Womens Health 2013; 5:9-17.

49. Berer M. Provision of abortion by mid-level providers: international policy, practice and perspectives. Bull World Health Organ 2009; 87:58-63.

50. Fórum Brasileiro de Segurança Pública. Anuário brasileiro de segurança pública, 2020. São Paulo: Fórum Brasileiro de Segurança Pública; 2020.

51. Instituto de Pesquisa Econômica Aplicada. Estupro no Brasil: uma radiografia segundo os dados da saúde. Brasília: Instituto de Pesquisa Econômica Aplicada; 2014. (Nota Técnica, 11).

52. Datafolha; Centro de Estudos de Criminalidade e Segurança Pública, Universidade Federal de Minas Gerais; Ministério da Justiça. Pesquisa Nacional de Vitimização. Brasília: Ministério da Justiça; 2013.
53. Delziovo CR, Coelho EBS, d'Orsi E, Lindner SR. Violência sexual contra a mulher e o atendimento no setor saúde em Santa Catarina Brasil. Ciênc Saúde Colet 2018; 23:1687-96.

54. Faúndes A, Rosas CF, Bedone AJ, Orozco LT. Violência sexual: procedimentos indicados e seus resultados no atendimento de urgência de mulheres vítimas de estupro. Rev Bras Ginecol Obstet 2006; 28:126-35.

55. Holmes MM, Resnick HS, Kilpatrick DG, Best CL. Rape-related pregnancy: estimates and descriptive characteristics from a national sample of women. Am J Obstet Gynecol 1996; 175:320-5

56. Chemlal S, Russo G. Why do they take the risk? A systematic review of the qualitative literature on informal sector abortions in settings where abortion is legal. BMC Womens Health 2019; 19:55.

57. Duarte NIG, Moraes LL, Andrade CB. A experiência do aborto na rede: análise de itinerários abortivos compartilhados em uma comunidade online. Ciênc Saúde Colet 2018; 23:3337-46.

58. Heilborn ML, Cabral CS, Brandão ER, Faro L, Cordeiro F, Azize RL. Itinerários abortivos em contextos de clandestinidade na cidade do Rio de Janeiro - Brasil. Ciênc Saúde Colet 2012; 17:1699-708.

59. Goes EF, Menezes GMS, Almeida MCC, Araújo TVB, Alves SV, Alves MTSSB, et al. Vulnerabilidade racial e barreiras individuais de mulheres em busca do primeiro atendimento pós-aborto. Cad Saúde Pública 2020; 36 Suppl 1:e00189618.

60. Lima NDF, Cordeiro RLM. "A minha vida não pode parar": itinerários abortivos de mulheres jovens. Revista Estudos Feministas 2020; 28:e58290.

61. Ministério da Saúde. Portaria no 415, de 21 de maio de 2014. Inclui o procedimento interrupção da gestação/antecipação terapêutica do parto previstas em lei e todos os seus atributos na Tabela de Procedimentos, Medicamentos, Órteses/Próteses e Materiais Especiais do SUS. Diário Oficial da União 2014; 22 mai.

62. Ministério da Saúde. Portaria no 437, de 28 de maio de 2014. Revoga as Portarias no 224/SAS/ MS, de 26 de março de 2014, 272/SAS/MS, de 2 de abril de 2014, 227/SAS/MS, de 4 de abril de 2014 e 415/SAS/MS, de 21 de maio de 2014. Diário Oficial da União 2014; 29 mai. 


\section{Abstract}

Abortion is supplied by the Brazilian Unified $\mathrm{Na}$ tional Health System (SUS) in cases allowed under the prevailing legislation. The current study seeks to map and characterize the supply and performance of this procedure in Brazil in 2019. Data included the Referral Services for Termination of Pregnancy Authorized by Law (SRIGCPL) recorded in the National Registry of Healthcare Establishments (SCNES) and the establishments with records of abortion for medical and legal reasons in the Outpatient Information System and Hospital Information System. Establishments were characterized by type and subtype, legal status, and contractual agreements, and georeferenced according to data from the SCNES. Next, municipalities were classified as those with and without supply of the procedure in 2019 and were presented by categories of Municipal Human Development Index (HDI-M) and population size. The data were used to calculate the rates of legally authorized abortions performed in the two groups of municipalities. In all, 290 establishments supplied the service, of which 101 SRIGCPL and 251 establishments with records of the procedure. The establishments were situated in 3.6\% (200) of Brazil's municipalities. The supply was mostly in hospitals (98.6\%), under the public administration (62.1\%), in contractual agreements with the SUS (99.7\%), in municipalities in the Southeast of Brazil (40.5\%), with more than 100,000 inhabitants (59.5\%), and with high or very high HDI-M (77.5\%). The rate of legally authorized abortions in childbearing-age residents of municipalities without supply of the service was 4.8 times lower than in municipalities with the service. The supply of legally authorized abortions in Brazil is distributed unequally across the territory, with possible negative implications for access to the service.

Health Services Accessibility; Legal Abortion; Equity in Access to Health Services; Reproductive Health Services

\section{Resumen}

En los casos previstos por la ley, el aborto es ofrecido por el Sistema Único de Salud brasileño (SUS). El presente estudio busca mapear y caracterizar la oferta y realización del procedimiento en Brasil en 2019. Se incluyeron los Servicios de Referencia para la Interrupción del Embarazo en los Casos Previstos por Ley (SRIGCPL), registrados en el Sistema del Registro Nacional de Establecimientos de Salud (SCNES), así como los establecimientos con registros de aborto por razones médicas y legales en el Sistema de Información Ambulatoria $o$ en el Sistema de Información Hospitalaria. Los establecimientos se caracterizaron por tipo y subtipo, naturaleza jurídica y convenios, y fueron georreferenciados a partir de los datos del SCNES. En seguida, los municipios se dividieron entre los que contaban con oferta y los que carecían de ella en 2019, entonces se presentaron por categorias de Índice de Desarrollo Humano Municipal (IDH-M) y tamaño poblacional. Luego se calculó la tasa de abortos previstos por ley de los dos grupos de municipios. En total, 290 establecimientos ofertaban el servicio, donde 101 eran SRIGCPL y 251 establecimientos con registro de procedimiento. Los establecimientos eran un 3,6\% (200) de los municipios brasileños. La oferta se produjo mayoritariamente en hospitales (98,6\%), de administración pública (62,1\%), con convenio del SUS (99,7\%), en municipios de la región Sudeste (40,5\%), con más de 100 mil habitantes (59,5\%) y de IDH-M alto o muy alto (77,5\%). La tasa de abortos previstos por ley entre las residentes en edad fértil de los municipios sin oferta del servicio fue de 4, 8 veces menor que en los municipios con este servicio. La oferta del aborto previsto por ley en Brasil se produce de forma desigual en el territorio, con una posible implicación en el acceso al servicio.

Accesibilidad a los Servicios de Salud; Aborto Legal; Equidad en el Acceso a los Servicios de Salud; Servicios de Salud Reproductiva
Recebido em 31/Mar/2021

Versão final reapresentada em 28/Mai/2021

Aprovado em 02/Jun/2021 